\title{
Short-term tidal variations in UT1: compliance between modelling and observation
}

\author{
Sigrid Englich, Harald Schuh \& Robert Weber \\ Institute of Geodesy and Geophysics, Vienna University of Technology, \\ Gusshausstrasse 27-29, 1040 Vienna, Austria \\ email: sigrid.englich@tuwien.ac.at
}

\begin{abstract}
The Earth rotation rate and consequently universal time (UT1) and length of day (LOD) are periodically affected by solid Earth tides and oceanic tides. Solid Earth tides induce changes with periods from around 5 days to 18.6 years, with the largest amplitudes occurring at fortnightly, monthly, semi-annual and annual periods, and at 18.6 years. The principal variations caused by oceanic tides have diurnal and semi-diurnal periods. For the investigation of the tidal effects with periods of up to 35 days, UT1 series are estimated from VLBI observation data of the time interval 1984-2008. The amplitudes and phases of the terms of interest are calculated and the results for diurnal and sub-diurnal periods are compared and evaluated with tidal variations derived from a GNSS-based LOD time series of 8 months. The observed tidal signals are finally compared to the predicted tidal variations according to recent geophysical models.
\end{abstract}

Keywords. UT1, tidal variations, VLBI, GNSS

\section{Diurnal and sub-diurnal variations}

We estimated the amplitudes of 70 ocean tidal terms with diurnal and semi-diurnal periods from VLBI/GNSS-observed UT1/LOD variations. The comparison with UT1 amplitudes of the conventional model (IERS Conventions (2004)), showed deviations in both tidal bands of up to $2.5 \mu \mathrm{s}$. The residuals in the semi-diurnal tidal band could be reduced almost to zero by accounting for the effect of the lunisolar torque on the triaxial Earth (Brzezinski \& Capitaine (2002)), to which the space geodetic techniques are sensitive.

\section{Variations up to 35 days}

The zonal tidal terms were derived from VLBI-based UT1 time series, from which the atmospheric influence and sub-diurnal variations were removed and all signal with periods over 35 days was filtered. All terms were calculated w.r.t. the IERS conventional model as well as w.r.t. a combination of different models following Gross (2009). The comparison with the IERS model revealed large discrepancies $(>40 \mu s)$ in the fortnigthly terms, whereas the combined model accounts for most of the fortnightly tidal signal.

\section{References}

Brzezinski, A. \& Capitaine, N. 2002, Proceedings of the Journees 2001, p. 51-58

Gross, R. S. 2009, Journal of Geodynamics, to be published in 2009

IERS Conventions 2003, in: D. D. McCarthy \& G. Petit (eds.), IERS Technical Note 32, 2004 